NBI-HE-97-08

February 1997

\title{
On the Connection Between 2d Topological Gravity and the Reduced Hermitian Matrix Model
}

\author{
J. Ambjørn!, M. G. Harris and M. Weis] \\ The Niels Bohr Institute, \\ Blegdamsvej 17, DK-2100 Copenhagen Ø, Denmark.
}

\begin{abstract}
We discuss how concepts such as geodesic length and the volume of space-time can appear in $2 \mathrm{~d}$ topological gravity. We then construct a detailed mapping between the reduced Hermitian matrix model and $2 \mathrm{~d}$ topological gravity at genus zero. This leads to a complete solution of the counting problem for planar graphs with vertices of even coordination number. The connection between multi-critical matrix models and multi-critical topological gravity at genus zero is studied in some detail.
\end{abstract}

PACS: 04.60.-m

Keywords: topological gravity; 2d quantum gravity; matrix model

\footnotetext{
${ }^{1}$ E-mail: ambjorn@nbi.dk

${ }^{2}$ E-mail: Martin.Harris@nbi.dk

${ }^{3}$ E-mail: weis@nbi.dk
} 


\section{Introduction}

Despite substantial progress in our understanding of two-dimensional quantum gravity we are still left with two separate theories: two-dimensional topological gravity and Liouville theory. Topological gravity is a theory where concepts such as geodesic distance make no sense. It can be viewed as a theory on the moduli space of punctured Riemann surfaces, and it is defined in such a way that it allows us to calculate intersection indices related to the moduli spaces. In Liouville theory geodesic distance plays a key rôle: Hartle-Hawking wave functions are defined in terms of the geodesic length of the boundaries [1], 2] and it is possible to consider the quantum theory of universes of fixed space-time volume, as well as correlation functions depending on the geodesic distance between two punctures [3] (see

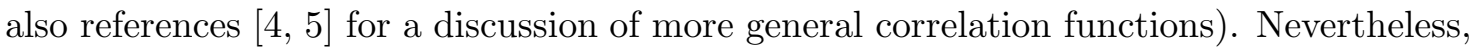
the two theories seem to be identical. The various proofs of the equivalence have so far been rather indirect [6, 7]. Typically, generalized Schwinger-Dyson equations have been derived for the two theories and have been shown to be identical by suitable identifications of variables. By such identifications the concepts of length and area enter into topological gravity without any obvious interpretation. As an example let us mention the following situation: in topological gravity we can calculate the expectation values $\left\langle\sigma_{n}\right\rangle$ of certain generalized puncture operators and we can define the generating function for this set of observables by

$$
\langle W(\ell)\rangle=\sum_{n} \frac{\ell^{n}}{n !}\left\langle\sigma_{n}\right\rangle .
$$

In topological gravity $\ell$ has no interpretation, beyond that of an indeterminate which defines the generating function. However, $\langle W(\ell)\rangle$ agrees with the corresponding HartleHawking wave functional calculated by the use of dynamical triangulations. In Liouville theory $\ell$ has from first principles an interpretation as the length of a macroscopic boundary of the two-dimensional universe. The same arguments apply to the total space-time volume $A$, which is defined from first principles if one uses Liouville theory, while it has no natural definition within the framework of topological gravity.

The rest of this paper is organized as follows: in section 2 we discuss how concepts such as the volume of space-time and the length of boundaries can be introduced in topological gravity. Since these concepts are natural in topological quantum gravity it should be possible to make a detailed mapping to Liouville theory. This is most conveniently done if we use the dynamically triangulated version of Liouville theory. In order to define the mapping we introduce in section 3 a contour integral representation of the partition function of topological gravity. A related integral representation for the Hermitian matrix model allows us in section 4 to perform a simple and detailed identification between the correlation functions in topological gravity and those in quantum gravity defined via dynamical triangulations. As a side result of this identification we use topological gravity 
results to solve completely the counting problem for planar graphs, whose vertices all have even coordination numbers. In section 5 we apply the mapping between topological gravity and matrix models to the case of multi-critical models. Section 6 contains our conclusions.

\section{Lagrangian Aspects of Topological Gravity}

Various starting points are possible in the case of topological gravity. One approach is to start directly from geometric considerations involving the intersection indices on punctured Riemann surfaces [8, 9]. This approach was completed and made mathematically rigorous by the seminal work of Kontsevich [10]. From the remarkable properties of the Kontsevich integral it follows that the partition function of topological gravity is a taufunction for the Korteweg-de Vries equation. More precisely, the partition function is given by the following matrix integral

$$
Z_{N}(M)=\frac{\int d \phi \exp \left\{-\operatorname{Tr}\left(\frac{M \phi^{2}}{2}+\frac{i \phi^{3}}{6}\right)\right\}}{\int d \phi \exp \left\{-\operatorname{Tr}\left(\frac{M \phi^{2}}{2}\right)\right\}},
$$

where the integration is over $N \times N$ Hermitian matrices $\phi$. The matrix $M$ is assumed to be symmetric and positive definite, and one can show that $Z_{N}(M)$ only depends on

$$
t_{k}=-\frac{(2 k-1) ! !}{k !} \operatorname{Tr} M^{-(2 k+1)}, \quad k \geq 0
$$

where by convention $(-1) ! !=1$. For $N \rightarrow \infty$ the $u$-function of the KdV-equation is then

$$
u\left(\left\{t_{i}\right\}\right)=\frac{\partial^{2}}{\partial t_{0}^{2}} \log Z\left(\left\{t_{i}\right\}\right) .
$$

One can write (for $N \rightarrow \infty$ ):

$$
\log Z\left(\left\{t_{i}\right\}\right)=\left\langle\exp \sum_{j=0}^{\infty} t_{j} \sigma_{j}\right\rangle=\sum_{n_{0}=0}^{\infty} \sum_{n_{1}=0}^{\infty} \cdots\left\langle\sigma_{0}^{n_{0}} \sigma_{1}^{n_{1}} \cdots\right\rangle \prod_{j=0}^{\infty}\left(\frac{t_{j}^{n_{j}}}{n_{j} !}\right) .
$$

This expression has a large $N$ expansion which is at the same time a genus expansion and it allows us in principle to calculate the numbers $\left\langle\sigma_{0}^{n_{0}} \sigma_{1}^{n_{1}} \cdots\right\rangle_{g}$, which have the interpretation as intersection indices for each genus.

A number of proofs [6] exist of the equivalence between topological gravity formulated via (2) and Liouville gravity formulated via the use of dynamical triangulations. As already mentioned the proofs of the equivalence between the theory defined by (2) and the theory of gravity defined via the matrix models corresponding to dynamical triangulations are rather indirect and leave no hints of how to define observables associated with

\footnotetext{
${ }^{4}$ We view here Liouville gravity and simplicial gravity as representing the same kind of quantum gravity, since observables referring directly to geometric objects (Hartle-Hawking wave functionals etc.) can be defined in both theories, and since all calculations in the two theories agree so far.
} 
geodesic distances in topological gravity. For this purpose it is more convenient to turn to the original formulation [11] of topological gravity, which originated from Witten's general approach to topological field theories [12]. Here the starting point for a topological theory is

$$
\mathcal{L}=0 .
$$

The non-trivial content of (6) comes by defining the space of variables and performing a corresponding gauge fixing of the local symmetries. In the case of gravity one can choose as the field variable the metric $g_{\mu \nu}$. The Lagrangian (6) is invariant under the most general variation

$$
\delta g_{\mu \nu}=\kappa_{\mu \nu}
$$

It is common to factor out explicitly the invariance under diffeomorphisms. If $\xi_{\mu}$ denotes an infinitesimal diffeomorphism and $r_{\mu}^{\lambda}$ a general $2 \times 2$ matrix, one can write

$$
\delta g_{\mu \nu}=\xi_{\mu ; \nu}+\xi_{\nu ; \mu}-\left(r_{\mu}^{\lambda} g_{\lambda \nu}+r_{\nu}^{\lambda} g_{\lambda \mu}\right)
$$

or, relating more closely to non-critical string theory,

$$
\delta g_{\mu \nu}=\xi_{\mu ; \nu}+\xi_{\nu ; \mu}-\frac{1}{2} g_{\mu \nu} \xi_{\lambda}^{\lambda}+g_{\mu \nu} \phi
$$

in decreasing order of generality (see 13, 14 for a discussion of the possible representations of (7)). Gauge fixing according to either (8) or (9) amounts to defining (6) to mean

$$
\mathcal{L}_{\mathrm{GF}}=s \cdot \Psi
$$

where $s$ is the BRST operator for the gauge fixed symmetries. For both gauge fixings (8) and (9) the split into diffeomorphisms and a residual shift is not unique, since there exist transformations different from the identity which leave $g_{\mu \nu}$ invariant. This leads to the well known ghosts for ghosts (super-ghosts) in $\mathcal{L}_{G F}$. In the following we will use (8) where the corresponding field content of the gauge fixed Lagrangian (10) is the two-dimensional metric $g_{\mu \nu}$, the gravitino field $\psi_{\mu \nu}$ (the ghost field of the shift), the ghost field $c^{\mu}$ of diffeomorphisms and the super-ghost field $\gamma^{\mu}$. In this representation, the action of the BRST operator $s$ is as follows [11],

$$
\begin{array}{ll}
s \cdot g_{\mu \nu}=£_{c} g_{\mu \nu}+\psi_{\mu \nu}, & s \cdot c^{\mu}=\frac{1}{2} £_{c} c^{\mu}+\gamma^{\mu}, \\
s \cdot \psi_{\mu \nu}=£_{c} \psi_{\mu \nu}-£_{\gamma} g_{\mu \nu}, & s \cdot \gamma^{\mu}=£_{c} \gamma^{\mu},
\end{array}
$$

where $£_{c}$ and $£_{\gamma}$ denote the action of infinitesimal diffeomorphisms with parameters $c^{\mu}$ and $\gamma^{\mu}$, and where $\psi_{\mu \nu}$ is related to the ghost $w_{\nu}^{\mu}$ for the matrix $r_{\nu}^{\mu}$ by

$$
\psi_{\mu \nu}=-w_{\mu}^{\gamma} g_{\gamma \nu}-w_{\nu}^{\gamma} g_{\gamma \mu}
$$


One obtains the observables starting from the Euler 2-form [15]

$$
\sigma^{(2)}=\frac{1}{8 \pi} \sqrt{g} R \varepsilon_{\mu \nu} d x^{\mu} \wedge d x^{\nu}
$$

which gives rise to a 1 -form and a 0 -form via the descent equations:

$$
s \cdot \sigma^{(2)}=d \sigma^{(1)}, \quad s \cdot \sigma^{(1)}=d \sigma^{(0)}, \quad s \cdot \sigma^{(0)}=0 .
$$

In this equation $s$ is the BRST operator and $d$ the exterior derivative. Explicitly one gets

$$
\begin{aligned}
\sigma^{(0)} & =\frac{\sqrt{g} \varepsilon_{\mu \nu}}{4 \pi}\left[\frac{1}{2} c^{\mu} c^{\nu} R+c^{\mu} D_{\rho}\left(\psi^{\nu \rho}-g^{\nu \rho} \psi_{\sigma}^{\sigma}\right)+D^{\mu} \gamma^{\nu}-\frac{1}{4} \psi_{\rho}^{\mu} \psi^{\nu \rho}\right] \\
\sigma^{(1)} & =\frac{\sqrt{g} \varepsilon_{\mu \nu}}{4 \pi}\left[c^{\nu} R+D_{\rho}\left(\psi^{\nu \rho}-g^{\nu \rho} \psi_{\sigma}^{\sigma}\right)\right] d x^{\mu}
\end{aligned}
$$

where $D_{\mu}$ denotes the covariant derivative. One can build an infinite set of observables from $\sigma^{(0)}$ since the super-ghost $\gamma^{\mu}$ is commutative:

$$
\sigma_{n}^{(0)} \equiv\left(\sigma^{(0)}\right)^{n}
$$

The expectation values of observables in topological gravity are now defined by functional integrals like

$$
\left\langle\prod_{k} \sigma_{n_{k}}\left(P_{k}\right)\right\rangle \equiv \int \mathcal{D} \Phi e^{-S[\Phi]} \prod_{k} \sigma_{n_{k}}\left(P_{k}\right),
$$

where $\Phi$ denotes a suitable collection of fields (see below) and the Lagrangian $\mathcal{L}$ associated with the action $S[\Phi]$ is BRST exact. The zero-forms $\sigma_{n_{k}}$ sit at the points $P_{k}$ on the world-sheet manifold and the super-gauge transformations should leave these points fixed. Correspondingly, the fields $c$ and $\gamma$ must vanish at these points and this implies that we consider punctured Riemann surfaces. In this way the Riemann surfaces considered in the path integral become a function of the observables. This is in accordance with the interpretation of the "observables" $\operatorname{Tr} \phi^{2 l_{1}} \ldots \operatorname{Tr} \phi^{2 l_{k}}$ in the Hermitian matrix model. The matrix integral corresponding to these observables is equivalent to a summation over (generalized) triangulations with $k$ boundaries of length $2 l_{1}, \ldots, 2 l_{k}$, i.e. for each matrix observable we consider different triangulated surfaces. For fixed $l_{1}, \ldots, l_{k}$ the continuum lengths of the boundaries go to zero and the loops become punctures. In later sections we will make the correspondence between the $\sigma_{n_{k}}$ 's and the matrix model observables precise.

In order to perform the functional integral (18) one usually chooses a background metric $g^{0}$ and background gravitino field $\psi^{0}$, which depend on the moduli of the punctured Riemann surfaces, and after successive integration over moduli all explicit reference to the points $P_{k}$ disappears [17]. In the following we will explicitly suppress all dependence 
on moduli as they play no rôle in our arguments. The background metric and background gravitino are introduced in a BRST exact way via Lagrange multiplier anti-ghosts $b^{\mu \nu}$ and $\beta_{\mu \nu}$, and their corresponding Nakanishi-Lautrup fields $d^{\mu \nu}$ and $\delta^{\mu \nu}$. The BRST algebra extends trivially to these new fields

$$
\begin{array}{ll}
s \cdot b^{\mu \nu}=d^{\mu \nu}, & s \cdot d^{\mu \nu}=0, \\
s \cdot \beta^{\mu \nu}=\delta^{\mu \nu}, & s \cdot \delta^{\mu \nu}=0 .
\end{array}
$$

The gauge fixed action is

$$
\begin{aligned}
S_{\mathrm{GF}} & =\int_{\Sigma} d^{2} x \sqrt{g} \mathcal{L}_{\mathrm{GF}}=\int_{\Sigma} d^{2} x \sqrt{g} s \cdot \Psi \\
& =\int_{\Sigma} d^{2} x \sqrt{g} s \cdot\left(b^{\mu \nu}\left(g_{\mu \nu}-g_{\mu \nu}^{0}\right)+\beta^{\mu \nu}\left(\psi_{\mu \nu}-\psi_{\mu \nu}^{0}\right)\right) .
\end{aligned}
$$

It is a remarkable fact that the functional integral (18) allows the extraction of almost complete information about the intersection indices on the moduli space of punctured Riemann surfaces. This is done by deriving Ward identities between various correlators and by showing that these Ward identities allow a recursive determination of expectation values of the $\sigma_{n}$ 's 116, 17].

Contrary to the Kontsevich integral formulation this definition of topological gravity operates with space-time, but offers a priori no concept of space-time distance. Sometimes it is even said that there should be no such concept in quantum gravity, since by reparameterization invariance, we only know if two points are separated or coincide and that this is the origin of the so-called contact terms. This is in contrast to the approach to quantum gravity via Liouville theory, or, at the constructive level, via dynamical triangulations. To be more explicit we can in Liouville theory define the partition function for a fixed space-time volume by

$$
Z_{\mathrm{LV}}(A)=\int \mathcal{D}[g] \delta\left(\int d^{2} \xi \sqrt{g}-A\right)
$$

and a Hartle-Hawking wave functional corresponding to a fixed boundary length $\ell$ and fixed space-time volume $A$ by:

$$
W_{\mathrm{LV}}(\ell, A)=\int \mathcal{D}[g] \delta\left(\int d^{2} \xi \sqrt{g}-A\right) \delta(\oint d s-\ell) .
$$

Similarly we can define the partition function of quantum universes with two marked points separated by a geodesic distance $R$ and with space-time volume $A$ by

$$
G_{\mathrm{LV}}(R, A)=\int \mathcal{D}[g] \delta\left(\int d^{2} \xi \sqrt{g}-A\right) \int d^{2} \xi \sqrt{g} \int d^{2} \xi^{\prime} \sqrt{g} \delta\left(D_{g}\left(\xi, \xi^{\prime}\right)-R\right),
$$

where $D_{g}\left(\xi, \xi^{\prime}\right)$ is the geodesic distance between $\xi$ and $\xi^{\prime}$ with respect to the metric $g$. In these equations the functional integration is over all equivalence classes of metrics. In a 
certain sense $Z_{\mathrm{LV}}(A), W_{\mathrm{LV}}(\ell, A)$ and $G_{\mathrm{LV}}(R, A)$ are "more topological" than topological gravity itself in the spirit of being a functional integral with $\mathcal{L}=0$, since no action is ever used in (21)-(23). However, at the same time they have explicit reference to metrical properties of the quantum space-time. In fact $G_{\mathrm{LV}}(R, A)$ is a perfect probe of the fractal structure of space-time [3]

We can also construct a partition function for topological gravity which corresponds to a fixed space-time volume. To do this we introduce the appropriate delta functions as a global, BRST exact term in the action,

$$
Z\left[A_{0}\right]=\int \mathcal{D} \Phi d \chi d \mu \exp \left\{-S_{\mathrm{GF}}-s \cdot\left[\chi\left(\int_{\Sigma} \sqrt{g}-A_{0}\right)\right]\right\}
$$

by use of Lagrange multipliers forming a constant anti-ghost multiplet $(\chi, \mu)$, which transforms like

$$
s \cdot \chi=\mu, \quad s \cdot \mu=0 .
$$

In contrast to equation (21) this also includes an additional delta function in the gravitino field, induced by supersymmetry. We must demand that the area of the surface is compatible with the chosen background metric, that is, $A_{0}=\int_{\Sigma} \sqrt{g_{0}}$. By Laplace transformation of (24) one finds a new topological action with a BRST exact "cosmological term",

$$
Z[\mu]=\int \mathcal{D} \Phi d \chi \exp \left\{-S_{\mathrm{GF}}-s \cdot\left(\chi \int_{\Sigma} \sqrt{g}\right)\right\}
$$

If the surface $\Sigma$ has boundaries it is also possible to introduce explicitly a "boundary cosmological term" living on the boundary of $\Sigma$ [18], and by an inverse Laplace transformation like (24) one can fix the length of the boundary and in this way obtain a topological gravity equivalent of (22)

The above considerations show that one can indeed define topological gravity on a restricted moduli space of metrics where for instance the area $A$ has a fixed value. In this way there is no conflict between the appearance of observables that refer to global metrical properties, such as the total area or the total length of a boundary, and the concept of topological gravity. We now proceed to establish a precise mapping between the operators $\sigma_{n}$ as they appear in topological gravity and the corresponding operators in the language of dynamical triangulations or matrix models.

\footnotetext{
${ }^{5}$ Strictly speaking it has not been possible to calculate $W_{\mathrm{LV}}(\ell, A)$ and $G_{\mathrm{LV}}(R, A)$ entirely within the continuum framework of Liouville theory. However, they can be calculated using dynamical triangulations 过, 2, 3.

${ }^{6}$ We believe that it is possible to define a topological gravity partition function corresponding to (23), too, but since we do not know how to calculate the corresponding partition function within the framework of topological gravity as a function of geodesic distance, we have not pursued this question further.
} 


\section{Topological Gravity at Genus Zero}

For the purpose of establishing this required map between topological gravity and matrix models we will consider genus zero surfaces only. Further it is simplest to start from the formulation of topological gravity found in 8 ].

\subsection{Contour Integral Representation}

In reference [8] Witten considers $2 \mathrm{~d}$ topological gravity with a perturbed action. The theory contains a set of basic operators, $\left\{\sigma_{n}\right\}$, where $n$ is an integer with $n \geq 0$, and the perturbation is parameterized by a set of coupling constants, $\left\{t_{n}\right\}$. The unperturbed correlation functions will be written with the subscript $V=0$; below we introduce a potential $V(z)$, which depends on the perturbed action, and $V=0$ corresponds to the unperturbed case. Then the partition function, $\langle 1\rangle_{V}$, for the perturbed theory at genus $g$ is given in terms of the unperturbed correlation functions for the same genus,

$$
\begin{aligned}
\langle 1\rangle_{V} & \equiv\left\langle\exp \left[\sum_{j=0}^{\infty} t_{j} \sigma_{j}\right]\right\rangle_{V=0} \\
& =\sum_{n_{0}=0}^{\infty} \sum_{n_{1}=0}^{\infty} \cdots\left(\prod_{j=0}^{\infty} \frac{t_{j}^{n_{j}}}{n_{j} !}\right)\left\langle\left(\sigma_{0}\right)^{n_{0}}\left(\sigma_{1}\right)^{n_{1}}\left(\sigma_{2}\right)^{n_{2}} \cdots\right\rangle_{V=0} .
\end{aligned}
$$

For genus zero the unperturbed correlation functions can be easily calculated from the puncture and dilaton equations [19]. With the normalization of $\sigma_{n}$ used in reference [8], we have,

$$
\left\langle\prod_{i=1}^{s} \sigma_{d_{i}}\right\rangle_{V=0}=\left(\sum_{i=1}^{s} d_{i}\right) ! \delta\left(3+\sum_{i=1}^{s}\left(d_{i}-1\right)\right),
$$

where $\delta(n) \equiv \delta_{n, 0}$. The correlation function of a product of observables for unperturbed topological gravity vanishes unless the total ghost number of the observables equals the dimension of the moduli space; the delta function reflects this fact. At higher genus the unperturbed correlation functions are much more complicated. The rest of this paper is almost exclusively concerned with genus zero surfaces and all the correlation functions given are for genus zero, unless it is stated otherwise.

Now we will define a potential $V(z)$ for the theory given in equation (27),

$$
V(z)=\sum_{j=0}^{\infty} t_{j} z^{j}
$$

where we have introduced a new complex variable $z$. We will write $t_{0}$ as $\epsilon$ and the puncture operator $\sigma_{0}$ as $P$, when it is convenient to do so. Then we can rewrite the 
partition function for the perturbed theory at genus zero as a contour integral. We have

$$
\begin{aligned}
& \langle 1\rangle_{V}=\sum_{n_{0}=0}^{\infty} \sum_{n_{1}=0}^{\infty} \cdots\left(\prod_{j=0}^{\infty} \frac{t_{j}^{n_{j}}}{n_{j} !}\right)\left(\sum_{k=0}^{\infty} k n_{k}\right) ! \delta\left(3+\sum_{k=0}^{\infty}(k-1) n_{k}\right) \\
& =\left[\left(1-\frac{\partial}{\partial \lambda}\right)^{-1} \sum_{n_{0}=0}^{\infty} \sum_{n_{1}=0}^{\infty} \cdots \prod_{j=0}^{\infty}\left(\frac{t_{j}^{n_{j}}}{n_{j} !} z^{(j-1) n_{j}} \lambda^{j n_{j}}\right)\right]_{\substack{\lambda=0 \\
\text { coeff. of } z^{-3}}},
\end{aligned}
$$

where

$$
\left(1-\frac{\partial}{\partial \lambda}\right)^{-1} \equiv \sum_{n=0}^{\infty}\left(\frac{\partial}{\partial \lambda}\right)^{n}
$$

The derivatives with respect to $\lambda$, give the factor of $\left(\sum k n_{k}\right)$ ! and keeping only the coefficient of $z^{-3}$ enforces the delta function. Thus,

$$
\langle 1\rangle_{V}=\left[\left(1-\frac{\partial}{\partial \lambda}\right)^{-1} \oint \frac{d z}{2 \pi i} z^{2} \exp \left(\frac{1}{z} V(\lambda z)\right)\right]_{\lambda=0},
$$

where we are integrating around some suitable contour encircling the origin. Changing variables $z \rightarrow z / \lambda$ gives

$$
\langle 1\rangle_{V}=\left[\left(1-\frac{\partial}{\partial \lambda}\right)^{-1} \oint \frac{d z}{2 \pi i} \frac{z^{2}}{\lambda^{3}} \exp \left(\frac{\lambda}{z} V(z)\right)\right]_{\lambda=0} .
$$

Expanding the exponential in powers of $\lambda$ and performing the differentiation with respect to $\lambda$ gives

$$
\langle 1\rangle_{V}=\sum_{r=3}^{\infty} \oint \frac{d z}{2 \pi i} z^{2}\left(\frac{V(z)}{z}\right)^{r} \frac{1}{r(r-1)(r-2)} .
$$

It is convenient to rescale $V$ by a factor of $\beta$; then after $V(z) \rightarrow \beta V(z)$ we have

$$
\frac{\partial^{3}}{\partial \beta^{3}}\langle 1\rangle_{\beta V}=\oint \frac{d z}{2 \pi i}\left(\frac{V^{3}}{z}\right) \frac{1}{1-\beta V / z} .
$$

Integrating with respect to $\beta$ three times and then setting $\beta=1$ gives

$$
\langle 1\rangle_{V}=-\frac{1}{2} \oint_{C} \frac{d z}{2 \pi i}(z-V)^{2} \log \left(\frac{z-V}{z}\right) .
$$

This formula, which gives the genus zero partition function for $2 \mathrm{~d}$ topological gravity with an arbitrary polynomial perturbation $V(z)$, will be used extensively throughout the rest of the paper. For a suitable choice of the parameters $\left\{t_{n}\right\}$, the integrand has a cut on the positive real axis from $z=0$ to some point, which we will call $z=u$. This point is defined by the equation $u=V(u)$, that is,

$$
u=\epsilon+\sum_{k=1}^{\infty} t_{k} u^{k}
$$

which is just the well-known string equation for genus zero [9]. The contour $C$ encircles this cut, across which the integrand has a discontinuity of $-2 \pi i$. It will be convenient to assume that $\epsilon \geq 0$. 


\subsection{Multi-Critical Models}

In this section we will calculate various correlation functions for the multi-critical models. The simplest choice of potential for the $k$-th multi-critical model is $V=\epsilon+z-z^{k}$, that is $t_{0} \neq 0, t_{1}=1$ and $t_{k}=-1$. Note however that this choice of potential is not unique and in section 5 we will show that the usual Hermitian one-matrix multi-critical models correspond to a different choice of $V(z)$. The scaling behaviour for the $k$-th multi-critical model is however independent of the particular choice of $V(z)$.

From $(38)$ we have that

$$
\langle 1\rangle=-\frac{1}{2} \oint_{C} \frac{d z}{2 \pi i}\left(z^{k}-\epsilon\right)^{2} \log \left(\frac{z^{k}-\epsilon}{z}\right),
$$

for the $k$-th multi-critical model. There is a cut on the real axis in the interval $\left[0, \epsilon^{1 / k}\right]$, which is encircled by the contour $C$. Thus,

$$
\langle 1\rangle=\frac{1}{2} \int_{0}^{\epsilon^{1 / k}} d x\left(x^{k}-\epsilon\right)^{2}=\frac{\epsilon^{2+\frac{1}{k}}}{\left(2+\frac{1}{k}\right)\left(1+\frac{1}{k}\right)},
$$

which agrees with the result in reference [8]. Note that the string susceptibility, $\gamma_{s t r}$, can be defined by $\langle 1\rangle \sim \epsilon^{2-\gamma_{s t r}}$. Thus we see that $\gamma_{s t r}=-\frac{1}{k}$ for the $k$-th multi-critical model.

The one-point correlation function can be obtained by differentiating (38) with respect to $t_{l_{1}}$. This gives for a general potential $V(z)$,

$$
\left\langle\sigma_{l_{1}}\right\rangle_{V}=\oint_{C} \frac{d z}{2 \pi i} z^{l_{1}}(z-V) \log \left(\frac{z-V}{z}\right) .
$$

Specializing to the $k$-th multi-critical model gives

$$
\left\langle\sigma_{l_{1}}\right\rangle=-\int_{0}^{\epsilon^{1 / k}} d x x^{l_{1}}\left(x^{k}-\epsilon\right)=\frac{\epsilon^{1+\left(l_{1}+1\right) / k}}{\left(l_{1}+1\right)\left(1+\left(l_{1}+1\right) / k\right)} .
$$

Similarly the two-point function is

$$
\left\langle\sigma_{l_{1}} \sigma_{l_{2}}\right\rangle_{V}=-\oint_{C} \frac{d z}{2 \pi i} z^{l_{1}+l_{2}} \log \left(\frac{z-V}{z}\right)=\int_{0}^{u} d x x^{l_{1}+l_{2}}=\frac{u^{l_{1}+l_{2}+1}}{\left(l_{1}+l_{2}+1\right)} .
$$

Hence for the multi-critical model,

$$
\left\langle\sigma_{l_{1}} \sigma_{l_{2}}\right\rangle=\frac{\epsilon^{\left(l_{1}+l_{2}+1\right) / k}}{\left(l_{1}+l_{2}+1\right)} .
$$

For the general potential we also have that $u=\langle P P\rangle_{V}$, a relationship which will prove useful later. The three-point function is

$$
\begin{aligned}
\left\langle\sigma_{l_{1}} \sigma_{l_{2}} \sigma_{l_{3}}\right\rangle_{V} & =\oint_{C} \frac{d z}{2 \pi i} \frac{z^{l_{1}+l_{2}+l_{3}}}{z-V} \\
& =\frac{\partial}{\partial \epsilon}\left[-\oint_{C} \frac{d z}{2 \pi i} z^{l_{1}+l_{2}+l_{3}} \log \left(\frac{z-V}{z}\right)\right]
\end{aligned}
$$


and in general,

$$
\left\langle\sigma_{l_{1}} \sigma_{l_{2}} \cdots \sigma_{l_{s}}\right\rangle_{V}=\left(\frac{\partial}{\partial \epsilon}\right)^{s-2}\left[-\oint_{C} \frac{d z}{2 \pi i} z^{L} \log \left(\frac{z-V}{z}\right)\right]=\left(\frac{\partial}{\partial \epsilon}\right)^{s-2}\left[\frac{u^{L+1}}{(L+1)}\right],
$$

where $L \equiv \sum_{i=1}^{s} l_{i}$. For the $k$-th multi-critical model, we put $u=\epsilon^{1 / k}$ in this formula. This result agrees with the calculation in references [8, 9] for the multi-critical models in topological gravity and also the similar result for the correlation functions in the multicritical Hermitian matrix model [20]; this latter model is studied in section 5 .

\subsection{Loop Operators}

In this section we define an operator $W(l)$, which we will call a loop operator, by analogy with similar observables defined in matrix models. The form of (42) suggests that we should define the loop operator as

$$
W(l)=\sum_{n=0}^{\infty} \frac{l^{n}}{n !} \sigma_{n}
$$

Let us now calculate expectation values of products of $W(l)$ for a general potential and for the multi-critical models. The results in the previous section immediately give for the potential $V(z)$

$$
\left\langle W\left(l_{1}\right)\right\rangle_{V}=\oint_{C} \frac{d z}{2 \pi i} e^{l_{1} z}(z-V) \log \left(\frac{z-V}{z}\right) .
$$

For the $k$-th multi-critical model this gives

$$
\left\langle W\left(l_{1}\right)\right\rangle=-\int_{0}^{\epsilon^{1 / k}} d x e^{l_{1} x}\left(x^{k}-\epsilon\right)=\left[\epsilon-\left(\frac{\partial}{\partial l_{1}}\right)^{k}\right]\left(\frac{e^{l_{1} \epsilon^{1 / k}}-1}{l_{1}}\right) .
$$

For $s \geq 2$,

$$
\begin{aligned}
\left\langle\prod_{i=1}^{s} W\left(l_{i}\right)\right\rangle_{V} & =\left(\frac{\partial}{\partial \epsilon}\right)^{s-2}\left[-\oint_{C} \frac{d z}{2 \pi i} \exp \left(z \sum_{i=1}^{s} l_{i}\right) \log \left(\frac{z-V}{z}\right)\right] \\
& =\left(\frac{\partial}{\partial \epsilon}\right)^{s-2}\left[\frac{e^{L u}-1}{L}\right],
\end{aligned}
$$

where $L=\sum_{i=1}^{s} l_{i}$ as before. The $k$-th multi-critical model is given by setting $u=\epsilon^{1 / k}$ in this last equation. The relationship between these loop operators and the corresponding correlators which occur in matrix models is discussed in section 4.4 . 


\subsection{The Dilaton and Puncture Equations}

The dilaton equation can be derived from (38) in a simple fashion; we have

$$
\sum_{k=0}^{\infty} t_{k} \frac{\partial}{\partial t_{k}}\langle 1\rangle_{V}=\oint_{C} \frac{d z}{2 \pi i} V(z-V) \log \left(\frac{z-V}{z}\right)
$$

and this gives us the dilaton equation,

$$
\left[\frac{\partial}{\partial t_{1}}-\sum_{k=0}^{\infty} t_{k} \frac{\partial}{\partial t_{k}}+2\right]\langle 1\rangle_{V}=0 .
$$

We will also derive the puncture equation. We have

$$
\left[\frac{\partial}{\partial \epsilon}-\sum_{k=0}^{\infty}(k+1) t_{k+1} \frac{\partial}{\partial t_{k}}\right]\langle 1\rangle_{V}=\oint_{C} \frac{d z}{2 \pi i}\left[\frac{d}{d z}(z-V)\right](z-V) \log \left(\frac{z-V}{z}\right) .
$$

After integrating by parts the right-hand side can be evaluated to give the puncture equation,

$$
\left[\frac{\partial}{\partial \epsilon}-\sum_{k=0}^{\infty}(k+1) t_{k+1} \frac{\partial}{\partial t_{k}}\right]\langle 1\rangle_{V}=\frac{1}{2} \epsilon^{2}
$$

Many of the results in these sections have been derived by different methods in a number of papers [8, 9, 16]. So far the contour integral representation of $2 \mathrm{~d}$ topological gravity, at genus zero, has allowed us to reproduce the formulae for various correlation functions in a very simple fashion. However, the real power of the method will become apparent when we consider the Hermitian one-matrix model in the next section.

\section{Hermitian Matrix Model}

\subsection{Contour Integral Representation}

Consider an Hermitian matrix model with an even potential; this is sometimes referred to as the reduced Hermitian matrix model. The partition function is defined as

$$
\overline{Z_{N}}(\bar{g})=\int \mathcal{D} M \exp [-\operatorname{Tr} \bar{U}]
$$

where $M$ is an $N \times N$ Hermitian matrix and the potential $\bar{U}$ is given by

$$
\bar{U}(M)=\frac{1}{2} M^{2}+\sum_{p=2}^{\infty} \frac{\overline{g_{p}}}{N^{p-1}} M^{2 p}
$$

Note that we are writing the variables with bars on them in order to differentiate them from the rescaled variables, which will be introduced in the next section. The free energy 
at genus zero is given by

$$
\overline{E_{0}}(\bar{g})=\lim _{N \rightarrow \infty}-\frac{1}{N^{2}} \log \left(\frac{\overline{Z_{N}}(\bar{g})}{\overline{Z_{N}}(0)}\right) .
$$

The problem of calculating $\overline{E_{0}}(\bar{g})$ has been solved using the technique of orthogonal polynomials and in reference 21] it is shown that

$$
\overline{E_{0}}(\bar{g})=\int_{0}^{a^{2}} d r w^{\prime}(r)[1-w(r)] \log \left(\frac{w(r)}{r}\right)
$$

where

$$
w(r)=r+\sum_{p=2}^{\infty} \overline{g_{p}} \frac{(2 p) !}{p !(p-1) !} r^{p}
$$

and $a^{2}$ is the solution of

$$
1=w\left(a^{2}\right), \quad a^{2}>0
$$

Integrating by parts gives

$$
\overline{E_{0}}(\bar{g})=\frac{1}{2} \int_{0}^{a^{2}} d r(1-w)^{2} \frac{d}{d r}\left[\log \left(\frac{w(r)}{r}\right)\right] .
$$

We would like to rewrite this equation as a contour integral in such a way as to make the connection with $2 \mathrm{~d}$ topological gravity clear. First let us change variables from the set of parameters $\left\{\overline{g_{p}}\right\}$ to the set $\left\{t_{p}\right\}$ by defining,

$$
\frac{\overline{g_{p}}(2 p) !}{p !(p-1) !}=-\frac{t_{p} \epsilon^{p-1}}{\left(1-t_{1}\right)^{p}} \quad \text { for } p \geq 2
$$

Note that the set of parameters $\left\{t_{p}\right\}$ has two more variables than the original set $\left\{\overline{g_{p}}\right\}$, namely $\epsilon$ and $t_{1}$; this gives us some freedom in the choice of these last two variables. When it is convenient we will write $\epsilon$ as $t_{0}$. In terms of these new parameters and using the variable $z$ in place of $r, w$ is given by

$$
w(z)-1=\frac{1}{\epsilon}\left[z^{\prime}-V\left(z^{\prime}\right)\right]
$$

where

$$
z^{\prime}=\frac{\epsilon z}{1-t_{1}}
$$

and $V(z)$ is defined as for topological gravity,

$$
V(z)=\sum_{p=0}^{\infty} t_{p} z^{p} .
$$

The integral in (64) is along the real axis from zero to $a^{2}$, where $a^{2}$ is given by the condition $w\left(a^{2}\right)=1$. This corresponds to the range $z^{\prime}=0$ to $z^{\prime}=u$, where $u$ is defined 
by $u=V(u)$. This is precisely where the cut occurs on the real axis for topological gravity with the potential $V$ and suggests that we should rewrite (64) as

$$
\overline{E_{0}}(\bar{g})=-\frac{1}{2} \oint \frac{d z}{2 \pi i}[1-w(z)]^{2}\left[\frac{d}{d z} \log \left(\frac{w(z)}{z}\right)\right] \log \left(\frac{w(z)-1}{z}\right) .
$$

The contour encircles the cut from $z=0$ to $z=a^{2}$, which has been introduced by adding the final logarithm in the integrand. Having rewritten $\overline{E_{0}}$ as a contour integral all that remains is to eliminate $w(z)$ in favour of $V(z)$, using (66),

$$
\overline{E_{0}}=-\frac{1}{2 \epsilon^{2}} \oint_{C} \frac{d z}{2 \pi i}(z-V)^{2}\left[\frac{d}{d z} \log \left(\frac{z-V+\epsilon}{z}\right)\right] \log \left(\frac{z-V}{z}\right),
$$

where the contour $C$ is around the cut $[0, u]$ on the real axis. This gives the free energy at genus zero for the reduced Hermitian matrix model, and clearly the result is very similar to the genus zero partition function of topological gravity, equation (38). Equation (65) relates the parameters in the two models.

\subsection{Rescaled Matrix Model}

The subsequent analysis will be simpler if we rescale the matrix in the matrix model, removing all the factors of $N^{1-p}$ and introducing a parameter in front of the quadratic term in the potential. We introduce a matrix $\Phi=M\left(1-t_{1}\right)^{-\frac{1}{2}} N^{-\frac{1}{2}}$, set $g_{p}=\overline{g_{p}}\left(1-t_{1}\right)^{p}$ for $p \geq 2$ and define $g_{1}=-\frac{1}{2} t_{1}$. Then after substituting into equations (58) and (59) we have

$$
Z_{N}(g)=\int \mathcal{D} \Phi \exp [-N \operatorname{Tr} U(\Phi)]
$$

where

$$
U(\Phi)=\frac{1}{2} \Phi^{2}+\sum_{p=1}^{\infty} g_{p} \Phi^{2 p}
$$

and the genus zero free energy is

$$
E_{0}(g)=\lim _{N \rightarrow \infty}-\frac{1}{N^{2}} \log \left(\frac{Z_{N}(g)}{Z_{N}(0)}\right) .
$$

The contour integral representation is now,

$$
E_{0}=\frac{1}{2} \log \left(1-t_{1}\right)-\frac{1}{2 \epsilon^{2}} \oint_{C} \frac{d z}{2 \pi i}(z-V)^{2}\left[\frac{d}{d z} \log \left(\frac{z-V+\epsilon}{z}\right)\right] \log \left(\frac{z-V}{z}\right),
$$

with $V(z)$ defined by $(68)$ as before. The relationship between the two sets of parameters is

$$
\frac{g_{p}(2 p) !}{p !(p-1) !}=-t_{p} \epsilon^{p-1} \quad \text { for } p \geq 1
$$


For a given $U(\Phi)$ we may freely choose $\epsilon$ (with $\epsilon \neq 0$ ) and then all the other $t_{p}$ are determined.

The relationship between the reduced Hermitian matrix model and topological gravity is simpler if we differentiate $E_{0}$ with respect to $t_{l}$. For $l \geq 1$,

$$
\frac{\partial E_{0}}{\partial t_{l}}=\frac{-\delta_{l, 1}}{2\left(1-t_{1}\right)}-\frac{1}{2 \epsilon^{2}} \oint_{C} \frac{d z}{2 \pi i}\left[2 z^{l-1}(z-V) \log \left(\frac{z-V}{z}\right)-\frac{z^{l-1}(z-V)^{2}}{z-V+\epsilon}\right],
$$

which we gain after differentiating under the integral sign, integrating by parts and dropping analytic terms in the integrand. However the last term in the integrand cancels with the first term on the right-hand side, giving

$$
\frac{\partial E_{0}}{\partial t_{l}}=-\frac{1}{\epsilon^{2}}\left\langle\sigma_{l-1}\right\rangle_{V}
$$

and hence for $l \geq 1$,

$$
\left\langle\sigma_{l-1}\right\rangle_{V}=\lim _{N \rightarrow \infty} \frac{\epsilon^{l+1}}{N} \frac{l !(l-1) !}{(2 l) !}\left\langle\operatorname{Tr} \Phi^{2 l}\right\rangle .
$$

This equation relates, at genus zero, the one-point correlation functions in topological gravity with correlation functions in the corresponding reduced Hermitian matrix model. Setting $l=1$ gives

$$
\langle P\rangle_{V}=\lim _{N \rightarrow \infty} \frac{1}{N} \frac{\epsilon^{2}}{2}\left\langle\operatorname{Tr} \Phi^{2}\right\rangle
$$

which shows that the correlation function for genus zero surfaces with a single puncture in topological gravity, is proportional to the partition function for triangulated surfaces with a marked link, with a suitable identification of the coupling constants in the two theories, that is, equation (75).

In a similar fashion, one can show by differentiating $E_{0}$ with respect to $\epsilon$ that,

$$
\epsilon^{3} \frac{\partial E_{0}}{\partial \epsilon}=-\sum_{l=1}^{\infty} l t_{l+1}\left\langle\sigma_{l}\right\rangle_{V}
$$

Equation $(78)$ can be generalized to multi-point correlators. Let us define a set of operators, $\left\{\Sigma_{l}\right\}$, in the matrix model as follows,

$$
\Sigma_{l}=N \epsilon^{l-1} \frac{l !(l-1) !}{(2 l) !} \operatorname{Tr}\left(\Phi^{2 l}\right) \quad \text { for } l \geq 1 .
$$

Then we have, by repeatedly differentiating (78) with respect to $t_{l_{i}}$,

$$
\left\langle\sigma_{l_{1}-1} \prod_{i=2}^{s} \sigma_{l_{i}}\right\rangle_{V}=\lim _{N \rightarrow \infty}\left(\frac{\epsilon}{N}\right)^{2}\left\langle\prod_{i=1}^{s} \Sigma_{l_{i}}\right\rangle_{\text {conn. }} \quad \text { for } l_{i} \geq 1
$$


where $\langle\cdots\rangle_{\text {conn. }}$ denotes the connected part of the expectation value in the matrix model; that is,

$$
\left\langle\Sigma_{l_{1}} \Sigma_{l_{2}}\right\rangle_{\text {conn. }} \equiv\left\langle\Sigma_{l_{1}} \Sigma_{l_{2}}\right\rangle-\left\langle\Sigma_{l_{1}}\right\rangle\left\langle\Sigma_{l_{2}}\right\rangle
$$

and so on.

Thus it is clear that the operator $\sigma_{l}$ in topological gravity corresponds to the operator $\Sigma_{l}$ in the matrix model, although the relationship is slightly different for the first operator. Note that this identification differs somewhat from the usual one in the literature 8 , 过, 22, 23], as many papers confuse $\sigma_{l}$ with an operator $O_{l}$, which is related to the $l$-th multi-critical model. This point will be discussed further in section 5 .

\subsection{Power Series Expansion}

In this section the connection between topological gravity and the Hermitian matrix model is used to write the genus zero free energy of the matrix model as a power series. It is simplest to begin with the version of the matrix model defined in section 4.1. Using the relationship,

$$
\overline{E_{0}}=E_{0}-\frac{1}{2} \log \left(1-t_{1}\right)
$$

and (77) we have

$$
\frac{\partial \overline{E_{0}}}{\partial t_{1}}=-\frac{1}{\epsilon^{2}}\langle P\rangle_{V}+\frac{1}{2\left(1-t_{1}\right)} .
$$

Rewriting the derivative on the left-hand side in terms of $\left\{\overline{g_{p}}\right\}$ gives

$$
\sum_{p=2}^{\infty} p \overline{g_{p}} \frac{\partial \overline{E_{0}}(\bar{g})}{\partial \overline{g_{p}}}=-\frac{\left(1-t_{1}\right)}{\epsilon^{2}}\langle P\rangle_{V}+\frac{1}{2} .
$$

However, $\langle P\rangle_{V}$ can be written as a power series; using equation (31), after summing over $n_{0}$ and differentiating with respect to $t_{0}$, we have

$$
\langle P\rangle_{V}=\sum_{n_{1}=0}^{\infty} \sum_{n_{2}=0}^{\infty} \cdots\left(\prod_{j=1}^{\infty} \frac{t_{j}^{n_{j}}}{n_{j} !}\right) \frac{t_{0}^{2+\sum_{k=2}^{\infty}(k-1) n_{k}}}{\left(2+\sum_{k=2}^{\infty}(k-1) n_{k}\right) !}\left(\sum_{k=1}^{\infty} k n_{k}\right) !
$$

But,

$$
\sum_{n_{1}=0}^{\infty} \frac{t_{1}^{n_{1}}}{n_{1} !}\left(\sum_{k=1}^{\infty} k n_{k}\right) !=\left(\sum_{k=2}^{\infty} k n_{k}\right) !\left(1-t_{1}\right)^{-1-\sum_{k=2}^{\infty} k n_{k}}
$$

and so,

$$
\frac{\left(1-t_{1}\right)}{\epsilon^{2}}\langle P\rangle_{V}=\sum_{n_{2}=0}^{\infty} \sum_{n_{3}=0}^{\infty} \cdots \prod_{j=2}^{\infty}\left[\frac{1}{n_{j} !}\left(\frac{t_{j} \epsilon^{j-1}}{\left(1-t_{1}\right)^{j}}\right)^{n_{j}}\right] \frac{\left(\sum_{k=2}^{\infty} k n_{k}\right) !}{\left(2+\sum_{k=2}^{\infty}(k-1) n_{k}\right) !}
$$


Thus we have

$$
\sum_{p=2}^{\infty} p \overline{g_{p}} \frac{\partial \overline{E_{0}}}{\partial \overline{g_{p}}}=-\sum_{\left\{n_{i}\right\}^{\prime}} \frac{\left(\sum_{k=2}^{\infty} k n_{k}\right) !}{\left(2+\sum_{k=2}^{\infty}(k-1) n_{k}\right) !} \prod_{p=2}^{\infty}\left[\frac{1}{n_{p} !}\left(-\frac{\overline{g_{p}}(2 p) !}{p !(p-1) !}\right)^{n_{p}}\right]
$$

where we are summing each $n_{i}$ (for $i=2,3,4, \cdots$ ) from 0 to $\infty$, but are excluding the case for which all the $n_{i}$ are zero. Integrating this equation yields

$$
\overline{E_{0}}(\bar{g})=-\sum_{\left\{n_{i}\right\}^{\prime}} \frac{\left(\left(\sum_{k=2}^{\infty} k n_{k}\right)-1\right) !}{\left(2+\sum_{k=2}^{\infty}(k-1) n_{k}\right) !} \prod_{p=2}^{\infty}\left[\frac{1}{n_{p} !}\left(-\frac{\overline{g_{p}}(2 p) !}{p !(p-1) !}\right)^{n_{p}}\right] .
$$

Note that for the special case in which only $\overline{g_{2}} \neq 0$, this reduces to

$$
\overline{E_{0}}\left(\overline{g_{2}}\right)=-\sum_{n=1}^{\infty} \frac{(2 n-1) !}{(n+2) ! n !}\left(-12 \overline{g_{2}}\right)^{n},
$$

which is the well-known formula counting planar $\Phi^{4}$ graphs [25]. Using (84) gives

$$
E_{0}(g)=-\sum_{\left\{n_{i}\right\}^{\prime}} \frac{\left(\left(\sum_{k=1}^{\infty} k n_{k}\right)-1\right) !}{\left(2+\sum_{k=2}^{\infty}(k-1) n_{k}\right) !} \prod_{p=1}^{\infty}\left[\frac{1}{n_{p} !}\left(-\frac{g_{p}(2 p) !}{p !(p-1) !}\right)^{n_{p}}\right],
$$

where the sum is now over all values of $n_{1}, n_{2}, \cdots$ from 0 to $\infty$, with them not all zero. This formula completely solves the problem of counting planar graphs, whose vertices all have even coordination numbers.

\subsection{Matrix Model Loop Correlators}

Let us define loop correlators for the reduced Hermitian matrix model (71) as follows,

$$
W\left(p_{1}\right)=\frac{1}{N} \sum_{k_{1}=0}^{\infty} \frac{\left\langle\operatorname{Tr} \Phi^{2 k_{1}}\right\rangle}{p_{1}^{2 k_{1}+1}}
$$

and for $s \geq 2$,

$$
W\left(p_{1}, \cdots, p_{s}\right)=N^{s-2} \sum_{k_{1}, \cdots, k_{s}=1}^{\infty} \frac{\left\langle\operatorname{Tr} \Phi^{2 k_{1}} \cdots \operatorname{Tr} \Phi^{2 k_{s}}\right\rangle_{\mathrm{conn} .}}{p_{1}^{2 k_{1}+1} \cdots p_{s}^{2 k_{s}+1}} .
$$

In fact, we will only be interested in the genus zero part of these functions, which we will denote $W_{0}\left(p_{1}, \cdots, p_{s}\right)$; these are gained by taking the limit $N \rightarrow \infty$. 
Using equation (78) we can write $W_{0}\left(p_{1}\right)$ in terms of expectation values for the corresponding topological model,

$$
W_{0}\left(p_{1}\right)=\frac{1}{p_{1}}+\sum_{k_{1}=1}^{\infty} \frac{\left(2 k_{1}\right) !}{k_{1} !\left(k_{1}-1\right) !} \frac{\left\langle\sigma_{k_{1}-1}\right\rangle_{V}}{p^{2 k_{1}+1} \epsilon^{k_{1}+1}} .
$$

This gives using (42),

$$
W_{0}\left(p_{1}\right)=\frac{1}{p_{1}}+\left(\frac{2}{p_{1}^{3} \epsilon^{2}}\right) \oint_{C} \frac{d z}{2 \pi i}\left(1-\frac{z}{z_{1}}\right)^{-\frac{3}{2}}(z-V) \log \left(\frac{z-V}{z}\right),
$$

where we have defined $z_{1}=\frac{1}{4} \epsilon p_{1}^{2}$. Integrating by parts gives

$$
W_{0}\left(p_{1}\right)=-\left(\frac{1}{p_{1} \epsilon}\right) \oint_{C} \frac{d z}{2 \pi i}\left(1-\frac{z}{z_{1}}\right)^{-\frac{1}{2}}\left(1-V^{\prime}\right) \log \left(\frac{z-V}{z}\right),
$$

where $V^{\prime}(z) \equiv \frac{d V}{d z}$. For example, in the case of the Gaussian model, $U(\Phi)=\frac{1}{2} \Phi^{2}$, we have that $V=\epsilon$ and integrating gives

$$
W_{0}\left(p_{1}\right)=\left(\frac{1}{p_{1} \epsilon}\right) \int_{0}^{u} d x\left(1-\frac{x}{z_{1}}\right)^{-\frac{1}{2}}=\frac{p_{1}}{2}\left[1-\sqrt{1-\frac{4}{p_{1}^{2}}}\right]
$$

in agreement with the known result [24].

The general loop correlator is given by

$$
W_{0}\left(p_{1}, \cdots, p_{s}\right)=\frac{d}{d U\left(p_{s}\right)} \cdots \frac{d}{d U\left(p_{2}\right)} W_{0}\left(p_{1}\right),
$$

where

$$
\frac{d}{d U(p)}=-\sum_{k=1}^{\infty} \frac{1}{p^{2 k+1}} \frac{\partial}{\partial g_{k}}=\sum_{k=1}^{\infty} \frac{1}{p^{2 k+1}} \frac{(2 k) !}{k !(k-1) !} \frac{1}{\epsilon^{k-1}} \frac{\partial}{\partial t_{k}} .
$$

Acting with this operator on the contour integral (97) gives for $s \geq 2$,

$$
W_{0}\left(p_{1}, \cdots, p_{s}\right)=-\frac{1}{\epsilon^{2}}\left(\prod_{i=1}^{s} \frac{2}{p_{i}^{3}}\right) \oint_{C} \frac{d z}{2 \pi i}\left[\prod_{i=1}^{s}\left(1-\frac{z}{z_{i}}\right)^{-\frac{3}{2}}\right] z^{s-1}\left(\frac{\partial}{\partial \epsilon}\right)^{s-2} \log \left(\frac{z-V}{z}\right),
$$

where

$$
z_{i}=\frac{1}{4} \epsilon p_{i}^{2}
$$

Note that $W_{0}\left(p_{1}, \cdots, p_{s}\right)$ does not depend on the value of $\epsilon$; this parameter in the mapping between the matrix model and topological gravity can be freely chosen. It is convenient to choose $\epsilon=4$. Then for $s=2$ the contour integral can be converted to a real integral and calculated giving

$$
W_{0}\left(p_{1}, p_{2}\right)=\frac{1}{2} \frac{1}{\left(p_{1}^{2}-p_{2}^{2}\right)^{2}}\left[p_{1}^{2} \sqrt{\frac{p_{2}^{2}-u}{p_{1}^{2}-u}}+p_{2}^{2} \sqrt{\frac{p_{1}^{2}-u}{p_{2}^{2}-u}}-2 p_{1} p_{2}\right]
$$


where $u$ is the solution of $u=V(u)$ for $\epsilon=4$ and we have assumed that $p_{1}, p_{2}>0$.

For $s \geq 3$,

$$
W_{0}\left(p_{1}, \cdots, p_{s}\right)=\frac{1}{\epsilon^{2}}\left(\prod_{i=1}^{s} \frac{2}{p_{i}^{3}}\right) \oint_{C} \frac{d z}{2 \pi i}\left[\prod_{i=1}^{s}\left(1-\frac{z}{z_{i}}\right)^{-\frac{3}{2}}\right] z^{s-1}\left(\frac{\partial}{\partial \epsilon}\right)^{s-3} \frac{1}{z-V},
$$

which is being integrated around the pole at $z=u$. This can be easily evaluated to give

$$
W_{0}\left(p_{1}, \cdots, p_{s}\right)=\left(\frac{1}{M} \frac{\partial}{\partial u}\right)^{s-3}\left(\frac{1}{u M} \prod_{i=1}^{s} \frac{u}{2\left(p_{i}^{2}-u\right)^{\frac{3}{2}}}\right),
$$

where $M(u)=\frac{1}{4}\left(1-V^{\prime}(u)\right)$ and again $\epsilon=4$. These formulae, which were calculated for the reduced Hermitian matrix model, are identical in form to those for the loop correlators of the complex matrix model [2], showing the close connection between these two models at genus zero.

Next we take inverse Laplace transforms of $W_{0}\left(p_{1}, \cdots, p_{s}\right)$ with respect to each of the variables $p_{i}^{2}$ to get a new set of loop operators,

$$
w_{0}\left(l_{1}\right)=\frac{1}{N} \sum_{n_{1}=0}^{\infty} \frac{l_{1}^{n_{1}-\frac{1}{2}}}{\Gamma\left(n_{1}+\frac{1}{2}\right)}\left\langle\operatorname{Tr} \Phi^{2 n_{1}}\right\rangle
$$

and for $s \geq 2$,

$$
w_{0}\left(l_{1}, \cdots, l_{s}\right)=N^{s-2} \sum_{n_{1}, \cdots, n_{s}=1}^{\infty} \frac{l_{1}^{n_{1}-\frac{1}{2}}}{\Gamma\left(n_{1}+\frac{1}{2}\right)} \cdots \frac{l_{s}^{n_{s}-\frac{1}{2}}}{\Gamma\left(n_{s}+\frac{1}{2}\right)}\left\langle\operatorname{Tr} \Phi^{2 n_{1}} \cdots \operatorname{Tr} \Phi^{2 n_{s}}\right\rangle_{\text {conn. }} .
$$

Taking inverse Laplace transforms of (102) gives

$$
w_{0}\left(l_{1}, \cdots, l_{s}\right)=-\left.2^{s-4} \oint_{C} \frac{d z}{2 \pi i}\left[\prod_{i=1}^{s} \frac{\sqrt{l_{i}} e^{z l_{i}}}{\Gamma\left(\frac{3}{2}\right)}\right] z^{s-1}\left(\frac{\partial}{\partial \epsilon}\right)^{s-2} \log \left(\frac{z-V}{z}\right)\right|_{\epsilon=4} .
$$

Hence for $s \geq 2$,

$$
w_{0}\left(l_{1}, \cdots, l_{s}\right)=\frac{4^{s-2}}{\left[\Gamma\left(\frac{1}{2}\right)\right]^{s}} \sqrt{l_{1} \cdots l_{s}}\left(\frac{\partial}{\partial L}\right)^{s-1}\left\langle W\left(l_{1}\right) \cdots W\left(l_{s}\right)\right\rangle_{\epsilon=4},
$$

where $L=\sum_{i=1}^{s} l_{i}$ as usual and the expectation value of loop operators on the right-hand side is evaluated for the potential $V(z)$ with $\epsilon=4$. The one loop correlator is

$$
w_{0}\left(l_{1}\right)=\frac{\sqrt{l_{1}}}{\Gamma\left(\frac{1}{2}\right)}\left[\frac{1}{l_{1}}+\frac{1}{4}\left\langle W\left(l_{1}\right)\right\rangle_{\epsilon=4}\right] .
$$


These equations relate the matrix model loop correlation functions to the topological gravity loop operators which were studied in section 3.3. Using the results in that section one can rewrite $(110)$ as

$$
w_{0}\left(l_{1}, \cdots, l_{s}\right)=\left.\frac{4^{s-2}}{\left[\Gamma\left(\frac{1}{2}\right)\right]^{s}} \sqrt{l_{1} \cdots l_{s}}\left(\frac{\partial}{\partial L}\right)^{s-1}\left(\frac{\partial}{\partial \epsilon}\right)^{s-2}\left[\frac{e^{L u}-1}{L}\right]\right|_{\epsilon=4} .
$$

It is worth noting that in these formulae $u$ is proportional to $\epsilon$. For example, consider the case of pure gravity, $U(\Phi)=\frac{1}{2} \Phi^{2}+g_{2} \Phi^{4}$. If we calculate the corresponding $V(z)$ then the equation $u=V(u)$ gives

$$
u=\frac{\epsilon}{24 g_{2}}\left[\sqrt{1+48 g_{2}}-1\right] .
$$

This can be inserted into the previous formula to give the multi-loop correlator for pure gravity in the reduced Hermitian matrix model.

\section{Multi-Critical Hermitian Matrix Models}

\subsection{Definition of the Matrix Model}

In this section we consider the multi-critical Hermitian matrix models and compare them with the corresponding models in topological gravity. The matrix model will be defined as

$$
Z_{N}=\int \mathcal{D} \Phi \exp \left[-\frac{N}{\epsilon} \operatorname{Tr} U(\Phi)\right]
$$

where the potential is

$$
U(\Phi)=\frac{1}{2} \Phi^{2}-\sum_{n=1}^{\infty} \overline{\mu_{n}} U_{n}(\Phi)
$$

and the potential $U_{n}(\Phi)$ corresponds to that for the $n$-th multi-critical model,

$$
\begin{aligned}
U_{1}(\Phi) & =\frac{1}{2} \Phi^{2} \\
U_{2}(\Phi) & =\Phi^{2}-\frac{1}{12} \Phi^{4} \\
& \vdots \\
U_{n}(\Phi) & =\sum_{p=1}^{n}(-1)^{p-1} \Phi^{2 p} \frac{n !(p-1) !}{(n-p) !(2 p) !}
\end{aligned}
$$

these potentials were derived in reference [20].

The relationship between this matrix model and topological gravity is basically the same as in section 4.2 , except that we have rescaled $\Phi$ by a factor of $\epsilon^{-\frac{1}{2}}$; that is, if for 
the given $U(\Phi)$, the variables $\left\{g_{p}\right\}$ are defined by equation (72), then the parameters in the topological model are given by

$$
t_{p}=-\frac{g_{p}(2 p) !}{p !(p-1) !} \quad \text { for } p \geq 1
$$

The potential for the topological model corresponding to the above matrix model is

$$
V(z)=\epsilon-\sum_{n=1}^{\infty} \overline{\mu_{n}}\left[(1-z)^{n}-1\right]
$$

and the $k$-th multi-critical model is gained by choosing $\epsilon \neq 0, \overline{\mu_{1}}=1$ and $\overline{\mu_{k}}=-1$; that is,

$$
V(z)=\epsilon+z+(1-z)^{k}-1 \equiv V_{k},
$$

which we have denoted $V_{k}$. Note that this is different from the potential usually chosen in topological gravity for the $k$-th multi-critical model, namely $V=\epsilon+z-z^{k}$; this potential, which has $t_{1}=1$, would give $g_{1}=-\frac{1}{2}$ and hence $U(\Phi)=g_{k} \Phi^{2 k}$. Lacking a $\Phi^{2}$ term in the exponential, this matrix model does not correspond to the usual picture of surfaces made by gluing together polygons. This is the source of much of the confusion that has arisen when comparing the multi-critical matrix and topological models.

\subsection{Correlation Functions in the Multi-Critical Topological Model}

We begin by calculating the correlation functions for the topological model with the potential $V_{k}$ given in equation (122). Then in the next section we consider the connection between these correlation functions and those in the matrix model.

For the $k$-th multi-critical model, $u$ is given by $u=V_{k}(u)$ and hence,

$$
u=1-(1-\epsilon)^{\frac{1}{k}}=1-(\Delta \epsilon)^{\frac{1}{k}},
$$

where we have defined $\Delta \epsilon \equiv 1-\epsilon$.

The partition function for the $k$-th multi-critical model is given by substituting $V_{k}(z)$ into (38),

$$
\langle 1\rangle_{V_{k}}=\frac{1}{2} \int_{0}^{u} d x\left(\Delta \epsilon-(1-x)^{k}\right)^{2}=-\frac{1}{2} \int_{1}^{(\Delta \epsilon)^{1 / k}} d y\left(\Delta \epsilon-y^{k}\right)^{2},
$$

where we have changed variables to $y=1-x$. Thus dropping the regular terms,

$$
\langle 1\rangle_{V_{k}} \sim \frac{-k^{2}}{(2 k+1)(k+1)}(\Delta \epsilon)^{2+\frac{1}{k}}
$$

this scales in the same fashion as (41), which was calculated for the $k$-th multi-critical model using the simpler potential. 
Next we define a set of operators, $\left\{O_{l}\right\}$,

$$
O_{l}=-\sum_{n=0}^{l}(-1)^{n}\left(\begin{array}{l}
l \\
n
\end{array}\right) \sigma_{n},
$$

which are chosen so that they scale in the correct fashion. The operator $O_{l}$ gives a factor of $-(1-z)^{l}$ in the contour integral and hence using (42),

$$
\left\langle O_{l}\right\rangle_{V_{k}}=-\int_{1}^{(\Delta \epsilon)^{1 / k}} d y y^{l}\left(\Delta \epsilon-y^{k}\right) \sim \frac{-k}{(l+k+1)(l+1)}(\Delta \epsilon)^{1+(l+1) / k}
$$

where we have dropped the regular terms in the last expression.

Using (48), the general case (for $s \geq 2$ ) is given by

$$
\begin{aligned}
\left\langle\prod_{i=1}^{s} O_{l_{i}}\right\rangle_{V_{k}} & =-\left(-\frac{\partial}{\partial \epsilon}\right)^{s-2} \int_{1}^{(\Delta \epsilon)^{1 / k}} d y y^{L} \\
& \sim-\left(\frac{\partial}{\partial \Delta \epsilon}\right)^{s-2}\left[\frac{1}{(L+1)}(\Delta \epsilon)^{(L+1) / k}\right],
\end{aligned}
$$

where $L=\sum_{i=1}^{s} l_{i}$.

Thus, up to regular terms, the scaling with respect to $\Delta \epsilon$ of correlation functions of $O_{l}$ for the potential, $V_{k}=\epsilon+z+(1-z)^{k}-1$, is the same as the scaling with respect to $\epsilon$ of correlation functions of $\sigma_{l}$ using the simpler potential, $V=\epsilon+z-z^{k}$; these were calculated in section 3.2. Note that some papers confuse $\sigma_{l}$ with $O_{l}$, however they are in fact related by equation (126).

\subsection{Correlation Functions in the Multi-Critical Matrix Model}

In reference [20], Gross and Migdal study correlation functions for the $k$-th multi-critical Hermitian matrix model at the spherical level. The correlators that they calculate scale in exactly the same fashion as those we derived in the previous section for topological gravity. It will be instructive to examine the connection between the two calculations.

In their paper, the free energy of the matrix model is $-\log Z_{N}$; the partition function for this model can be written as in equation (114). After dropping a number of terms, which they claim do not contribute to the critical behaviour, the free energy is given by $F$, where

$$
\frac{\partial^{2} F}{\partial t^{2}}=f(t)
$$

and $f\left(t,\left\{\mu_{n}\right\}\right)$ is the relevant solution of

$$
t=f^{k}-\sum_{n=0}^{\infty} \mu_{n} f^{n} .
$$


We will take the last two equations as being the definition of $F$ and then later consider the connection between $F$ and the free energy of the matrix model. The correlation functions are defined as

$$
-\left.\frac{\partial}{\partial \mu_{l_{1}}} \cdots \frac{\partial}{\partial \mu_{l_{s}}} F\left(t,\left\{\mu_{n}\right\}\right)\right|_{\left\{\mu_{n}=0\right\}} .
$$

It should be noted that they introduced various powers of $N$ in their definitions of the scaling variables: $t, f(t)$ and so on. However these factors of $N$ do not affect the following arguments and can safely be ignored.

To make the connection with topological gravity we consider the potential,

$$
V(z)=-t+z+(1-z)^{k}-\sum_{n=0}^{\infty} \mu_{n}(1-z)^{n} .
$$

This is just equation (121) with the change of variables,

$$
\overline{\mu_{n}}=\mu_{n}+\delta_{n, 1}-\delta_{n, k}
$$

and

$$
\epsilon=1-t-\sum_{n=0}^{\infty} \mu_{n}
$$

The potential gives the $k$-th multi-critical model perturbed by terms corresponding to the operators $O_{n}$. Then the equation $u=V(u)$ can be written,

$$
t=(1-u)^{k}-\sum_{n=0}^{\infty} \mu_{n}(1-u)^{n} .
$$

Thus we see that $f=1-u$, where $u=\langle P P\rangle$ in this topological model as was shown in section 3.2. However $\frac{\partial}{\partial \epsilon}=-\frac{\partial}{\partial t}$, hence,

$$
\frac{\partial^{2} F}{\partial t^{2}}=f=1-\langle P P\rangle=1-\frac{\partial^{2}}{\partial t^{2}}\langle 1\rangle
$$

Integrating, we have that $F=-\langle 1\rangle$, up to some regular terms which we will drop. Thus $F$, which is supposed to scale in the same way as the free energy of the matrix model, is in fact proportional to the partition function of the topological model defined in (133), up to some regular terms. Noting that $t=\Delta \epsilon$, when $\mu_{n}=0$ for all $n$, then,

$$
-\left.\frac{\partial}{\partial \mu_{l_{1}}} \cdots \frac{\partial}{\partial \mu_{l_{s}}} F\right|_{\left\{\mu_{n}=0\right\}}=\left\langle O_{l_{1}} \cdots O_{l_{s}}\right\rangle_{V_{k}},
$$

since deriving with respect to $\mu_{l}$ just pulls down an $O_{l}$ operator. Note also that the correlation function on the right-hand side is calculated in topological gravity for the $k$-th multi-critical model (we calculated this in the previous section), it is not a matrix model correlator. Thus Gross and Migdal have been calculating topological correlation functions in their paper. The crucial question to answer is the following one: "If $F$ in equation 
(132) is replaced by the free energy of the matrix model, do the correlation functions still scale in the same fashion?" The answer is "Yes, provided that $F$ is replaced by $\epsilon^{2} E_{0}$." The factor of $\epsilon^{2}$ in front of the free energy is necessary, as without it there are extra terms in the correlation functions, which would change the scaling behaviour if any of the $l_{i}$ were greater than $k$.

The free energy of the matrix model, $E_{0}$, is defined as in equation (73). We wish to calculate the correlation functions, which are derivatives with respect to $\mu_{l_{i}}$ of $-\epsilon^{2} E_{0}$, evaluated at the $k$-th multi-critical model (that is, $\mu_{n}=0$ for all $n$ ). However we know from (126) that

$$
\frac{\partial}{\partial \mu_{l}}=\sum_{n=0}^{l}(-1)^{n+1}\left(\begin{array}{l}
l \\
n
\end{array}\right) \frac{\partial}{\partial t_{n}} .
$$

Using equations $(77)$ and $(80)$ one can calculate the effect of this operator on $\epsilon^{2} E_{0}$; note that these equations were left unchanged under the rescaling of $\Phi$ by $\epsilon^{-\frac{1}{2}}$, which we performed earlier. After some algebra, we have

$$
\left.\frac{\partial}{\partial \mu_{l}}\left(\epsilon^{2} E_{0}\right)\right|_{\left\{\mu_{n}=0\right\}}=-\epsilon \log k+\int_{1}^{(\Delta \epsilon)^{1 / k}} d y\left(\Delta \epsilon-y^{k}\right)\left[\frac{y^{l}}{1-y}-\frac{k y^{k-1}}{1-y^{k}}\right] .
$$

The leading singular behaviour is given by

$$
-\left.\frac{\partial}{\partial \mu_{l}}\left(\epsilon^{2} E_{0}\right)\right|_{\left\{\mu_{n}=0\right\}} \sim \frac{-k}{(l+k+1)(l+1)}(\Delta \epsilon)^{1+(l+1) / k} \sim\left\langle O_{l}\right\rangle_{V_{k}},
$$

as expected.

The scaling of $E_{0}$ can also be calculated from (141) since,

$$
\left.\frac{\partial}{\partial \epsilon}\left(\epsilon^{2} E_{0}\right)\right|_{\left\{\mu_{n}=0\right\}}=-\left.\frac{\partial}{\partial \mu_{0}}\left(\epsilon^{2} E_{0}\right)\right|_{\left\{\mu_{n}=0\right\}} \sim \frac{-k}{(k+1)}(\Delta \epsilon)^{1+\frac{1}{k}}
$$

and hence as expected,

$$
\left.\epsilon^{2} E_{0}\right|_{\left\{\mu_{n}=0\right\}} \sim \frac{k^{2}}{(2 k+1)(k+1)}(\Delta \epsilon)^{2+\frac{1}{k}} \sim-\langle 1\rangle_{V_{k}} .
$$

In principle one could extend this calculation to multi-point correlation functions, however the algebra rapidly becomes tedious. In order to prove that derivatives of $\epsilon^{2} E_{0}$ have the same leading singular behaviour as derivatives of $F$, it is convenient to return to reference [21]. This paper gives an alternative form of equation (61),

$$
\overline{E_{0}}(\bar{g})=-\int_{0}^{1} d x(1-x) \log \left(\frac{r_{0}(x)}{x}\right),
$$

where $r_{0}(x)$ is defined by $x=w\left(r_{0}\right)$. This leads to

$$
E_{0}(g)=\frac{1}{2} \log \epsilon-\frac{1}{\epsilon^{2}} \int_{0}^{\epsilon} d x(\epsilon-x) \log u(x),
$$


where $u(x)$ is the solution of $V(u(x))=u(x)$ for the potential (68) with $t_{0}=x$. Thus,

$$
\frac{\partial^{2}}{\partial \epsilon^{2}}\left(\epsilon^{2} E_{0}\right)=\frac{3}{2}+\log \epsilon-\log u(\epsilon)
$$

Dropping the first two terms, which are regular in $\Delta \epsilon$, we can differentiate with respect to $\mu_{l_{1}}$,

$$
\frac{\partial^{2}}{\partial \epsilon^{2}}\left[\frac{\partial}{\partial \mu_{l_{1}}}\left(\epsilon^{2} E_{0}\right)\right] \sim-\frac{\left\langle P P O_{l_{1}}\right\rangle}{\langle P P\rangle} \sim-\left\langle P P O_{l_{1}}\right\rangle
$$

where the last expression uses the fact that, $u(\epsilon)=\langle P P\rangle=1-(\Delta \epsilon)^{1 / k}$ and we are keeping only the leading non-analytic term. Integrating gives, up to regular and sub-leading terms,

$$
-\left.\frac{\partial}{\partial \mu_{l_{1}}}\left(\epsilon^{2} E_{0}\right)\right|_{\left\{\mu_{n}=0\right\}} \sim\left\langle O_{l_{1}}\right\rangle_{V_{k}} .
$$

This can easily be generalized to give

$$
-\left.\frac{\partial}{\partial \mu_{l_{1}}} \cdots \frac{\partial}{\partial \mu_{l_{s}}}\left(\epsilon^{2} E_{0}\right)\right|_{\left\{\mu_{n}=0\right\}} \sim\left\langle O_{l_{1}} \cdots O_{l_{s}}\right\rangle_{V_{k}} .
$$

Thus as claimed earlier, the correlation functions for the reduced Hermitian matrix model scale in the same fashion as the topological correlation functions, for the $k$-th multi-critical model.

It should perhaps be noted at this point that if one rescales all the variables by various powers of $N$ as in reference [20], then in the spherical limit $N \rightarrow \infty$ all the sub-leading terms in (149) vanish (this rescaling works provided we have dropped the regular terms). However, there is a simpler way of removing the sub-leading terms. From equation (77) we have that

$$
-\left(\frac{\partial}{\partial \mu_{l}}-\frac{\partial}{\partial \mu_{l+1}}\right)\left(\epsilon^{2} E_{0}\right)=\left\langle O_{l}\right\rangle_{V}
$$

which is true for a generic potential $V(z)$. Differentiating repeatedly and evaluating for $V_{k}$ gives

$$
-\left.\left(\frac{\partial}{\partial \mu_{l_{1}}}-\frac{\partial}{\partial \mu_{l_{1}+1}}\right) \frac{\partial}{\partial \mu_{l_{2}}} \cdots \frac{\partial}{\partial \mu_{l_{s}}}\left(\epsilon^{2} E_{0}\right)\right|_{\left\{\mu_{n}=0\right\}}=\left\langle O_{l_{1}} \cdots O_{l_{s}}\right\rangle_{V_{k}} .
$$

This equation is exact and there are no sub-leading terms.

Using (139) and (120), one can write $\frac{\partial}{\partial \mu_{l}}$ in terms of derivatives with respect to the variables $\epsilon$ and $g_{p}$,

$$
\frac{\partial}{\partial \mu_{l}}=\sum_{p=1}^{l}(-1)^{p} \frac{l !(p-1) !}{(l-p) !(2 p) !} \frac{\partial}{\partial g_{p}}-\frac{\partial}{\partial \epsilon} .
$$

In principle one can use this to operate on $\epsilon^{2} E_{0}$ and hence rewrite the correlation functions (151) as matrix model expectation values. The summation in 152 brings down a term proportional to $\operatorname{Tr} U_{l}(\Phi)$, but the derivative with respect to $\epsilon$ is more complicated and 
gives a number of terms including one proportional to $\operatorname{Tr} U(\Phi)$ in the expectation value. As a result the multi-point correlators are not particularly simple. Calculating the onepoint correlation function is however straightforward,

$$
\left\langle O_{l}\right\rangle_{V}=-\left(\frac{\partial}{\partial \mu_{l}}-\frac{\partial}{\partial \mu_{l+1}}\right)\left(\epsilon^{2} E_{0}\right)=\lim _{N \rightarrow \infty} \frac{\epsilon}{N}\left\langle\operatorname{Tr}\left[U_{l}(\Phi)-U_{l+1}(\Phi)\right]\right\rangle .
$$

In fact, defining

$$
\begin{aligned}
& \widetilde{U}_{l} \equiv \operatorname{Tr}\left[U_{l}(\Phi)-U_{l+1}(\Phi)\right], \\
& \bar{U}_{l} \equiv \operatorname{Tr}\left[U_{l}(\Phi)-\frac{1}{\epsilon} U(\Phi)\right]
\end{aligned}
$$

and

$$
\frac{\partial}{\partial \widetilde{\mu}_{l}} \equiv \frac{\partial}{\partial \mu_{l}}-\frac{\partial}{\partial \mu_{l+1}}
$$

we have for the first few multi-point correlation functions in the spherical limit $N \rightarrow \infty$,

$$
\begin{gathered}
\left\langle O_{l_{1}} O_{l_{2}}\right\rangle_{V}=-\frac{\partial}{\partial \widetilde{\mu_{l_{1}}}} \frac{\partial}{\partial \mu_{l_{2}}}\left(\epsilon^{2} E_{0}\right)=\left\langle\widetilde{U}_{l_{1}} \bar{U}_{l_{2}}\right\rangle_{\text {conn. }}-\frac{1}{N}\left\langle\widetilde{U}_{l_{1}}\right\rangle, \\
\left\langle O_{l_{1}} O_{l_{2}} O_{l_{3}}\right\rangle_{V}=-\frac{\partial}{\partial \widetilde{\mu_{1}}} \frac{\partial}{\partial \mu_{l_{2}}} \frac{\partial}{\partial \mu_{l_{3}}}\left(\epsilon^{2} E_{0}\right)=\left(\frac{N}{\epsilon}\right)\left\langle\widetilde{U}_{l_{1}} \bar{U}_{l_{2}} \bar{U}_{l_{3}}\right\rangle_{\text {conn. }}
\end{gathered}
$$

and

$$
\begin{aligned}
\left\langle O_{l_{1}} O_{l_{2}} O_{l_{3}} O_{l_{4}}\right\rangle_{V}= & \left(\frac{N}{\epsilon}\right)^{2}\left[\left\langle\widetilde{U}_{l_{1}} \bar{U}_{l_{2}} \bar{U}_{l_{3}} \bar{U}_{l_{4}}\right\rangle_{\text {conn. }}\right. \\
& \left.+\frac{1}{N}\left\langle\widetilde{U}_{l_{1}}\left(\bar{U}_{l_{2}} \bar{U}_{l_{3}}+\bar{U}_{l_{3}} \bar{U}_{l_{4}}+\bar{U}_{l_{4}} \bar{U}_{l_{2}}\right)\right\rangle_{\text {conn. }}\right] .
\end{aligned}
$$

The correlation functions in topological gravity and the Hermitian matrix model can be related more simply at the cost of reintroducing sub-leading terms. If we define,

$$
\widetilde{O}_{l}=O_{l}-O_{l+1}
$$

then in the spherical limit we have

$$
\left\langle O_{l_{1}}\right\rangle_{V}=\left(\frac{\epsilon}{N}\right)\left\langle\widetilde{U}_{l_{1}}\right\rangle
$$

and in general,

$$
\left\langle O_{l_{1}} \prod_{i=2}^{s} \widetilde{O}_{l_{i}}\right\rangle_{V}=\left(\frac{N}{\epsilon}\right)^{s-2}\left\langle\prod_{i=1}^{s} \widetilde{U}_{l_{i}}\right\rangle_{\text {conn. }} .
$$

This completes our study of the connection between the correlation functions in the reduced Hermitian matrix model and those in topological gravity. 


\section{Conclusion}

We have argued that it is possible in $2 \mathrm{~d}$ topological gravity to consider observables depending on geodesic distances, such as the volume of space-time and the length of the boundaries. We then constructed a detailed mapping between topological gravity and the reduced Hermitian matrix model, both for operators and coupling constants. In particular, the operators in topological gravity were associated with very specific matrix model operators even before the continuum limit of the matrix model was taken. As a spin-off of this identification we could use results from topological gravity to provide the complete solution of the counting problem for planar graphs whose vertices have all even coordination numbers. It is an interesting problem to consider whether these counting results can be extended to arbitrary genus. Further, the simple and exact relation between a set of planar graphs and the partition function of topological gravity hints that there may be a simpler interpretation of the intersection indices on the moduli spaces of Riemann surfaces with punctures. However, a substantiation of such a claim requires first a generalization of the results of this paper to surfaces of arbitrary genus. An alternative representation of the operators $\sigma_{n}$ might allow us to understand one remaining puzzle in topological gravity, namely the transition from local operators $\sigma_{n}$, which create only punctures in the Riemann surfaces, to operators which create macroscopic boundaries. Using matrix models such a transition is conceptionally simple since $\operatorname{Tr} \Phi^{2 n}$ will create a macroscopic boundary, if $n \rightarrow \infty$ as the square root of the (discretized) volume. Since we have shown

that $\left\langle\sigma_{n-1}\right\rangle$ is closely related to $\operatorname{Tr} \Phi^{2 n}$, the possibility of a geometric interpretation of $\left\langle\sigma_{n-1}\right\rangle$ for $n \rightarrow \infty$ should exist. We hope to be able to return to this question in a later publication.

\section{Acknowledgements}

MGH would like to acknowledge the support of the European Union through their TMR Programme. MW acknowledges the support of the Danish Natural Science Research Council (grant 11-0801).

\section{References}

[1] F. David, Mod. Phys. Lett. A5 (1990) 1019.

J. Ambjørn and Y. Makeenko, Mod. Phys. Lett. A5 (1990) 1753.

[2] J. Ambjørn, J. Jurkiewicz and Y. M. Makeenko, Phys. Lett. B251 (1990) 517. 
[3] J. Ambjørn and Y. Watabiki, Nucl. Phys. B445 (1995) 129.

J. Ambjørn, J. Jurkiewicz and Y. Watabiki, Nucl. Phys. B454 (1995) 313.

[4] H. Kawai, N. Kawamoto, T. Mogami and Y. Watabiki, Phys. Lett. B306 (1993) 19.

[5] H. Aoki, H. Kawai, J. Nishimura and A. Tsuchiya, Nucl. Phys. B474 (1996) 512.

[6] A. Gerasimov, Y. Makeenko, A. Marshakov, A. Mironov, A. Morozov and A. Orlov, Mod. Phys. Lett. A6 (1991) 3079.

A. Marshakov, A. Mironov and A. Morozov, Mod. Phys. Lett. A7 (1992) 1345; Phys. Lett. B274 (1992) 280.

S. Kharchev, A. Marshakov, A. Mironov, A. Morozov and A. Zabrodin, Nucl. Phys. B380 (1992) 181.

D. Gross and M. J. Newman, Nucl. Phys. B380 (1992) 168.

J. Ambjørn and C. F. Kristjansen, Mod. Phys. Lett. A8 (1993) 2875.

[7] R. Dijkgraaf, H. Verlinde and E. Verlinde, Nucl. Phys. B348 (1991) 435.

[8] E. Witten, Nucl. Phys. B340 (1990) 281.

[9] R. Dijkgraaf and E. Witten, Nucl. Phys. B342 (1990) 486.

[10] M. L. Kontsevich, Funkt. Anal. Pril. 25 (1991) 50.

C. Itzykson and J.-B. Zuber, Int. J. Mod. Phys. A7 (1992) 5661.

[11] J. M. F. Labastida, M. Pernici and E. Witten, Nucl. Phys. B310 (1988) 611.

[12] E. Witten, Int. J. Mod. Phys. A6 (1991) 2775.

[13] M. Henneaux and C. Teitelboim, Phys. Lett. B234 (1990) 487.

[14] S. Cordes, G. Moore and S. Ramgoolam, "Lectures on 2D Yang-Mills Theory, Equivariant Cohomology and Topological Field Theories," Les Houches lectures 1994, hepth/9411210.

[15] C. M. Becchi, R. Collina and C. Imbimbo, Phys. Lett. B322 (1994) 79.

[16] E. Verlinde and H. Verlinde, Nucl. Phys. B348 (1991) 457.

[17] C. Becchi and C. Imbimbo, Nucl. Phys. B462 (1996) 571.

[18] J. Hughes and D. Montano, Phys. Lett. B250 (1990) 33.

[19] R. Dijkgraaf, "Intersection Theory, Integrable Hierarchies and Topological Field Theory," lectures at Cargèse Summer School 16-27th July 1991, hep-th/9201003.

[20] D. J. Gross and A. A. Migdal, Nucl. Phys. B340 (1990) 333. 
[21] D. Bessis, C. Itzykson and J. B. Zuber, Adv. Appl. Math. 1 (1980) 109.

[22] I. R. Klebanov and R. B. Wilkinson, Nucl. Phys. B354 (1991) 475.

[23] J. Distler, Nucl. Phys. B342 (1990) 523.

[24] J. Ambjørn, Class. Quantum Grav. 12 (1995) 2079.

[25] E.Brézin, C.Itzykson, G.Parisi and J.B.Zuber, Commun. Math. Phys. 59 (1978) 35. 